\title{
Targeted cognitive behavioural therapy may reduce relapse in people with prodromal symptoms of schizophrenia
}

Gumley A, O' Grady M, McNay L et al. Early intervention for relapse in schizophrenia: results of a 12-month randomized controlled trial of cognitive behavioural therapy. Psychol Med 2003; 33: 419-431.

\section{QUESTION: Does cognitive behavioural therapy prevent relapse of schizophrenia compared with usual care in people with prodromal symptoms?}

\section{Design}

Randomised controlled trial.

\section{Setting}

8 community mental health teams, Scotland; June 1997 to December 2000.

\section{Participants}

144 people with schizophrenia or related disorder, fulfilling DSM-IV criteria, aged 18-65 years and at risk of relapse (history of relapse in the last 2 years, stressful or isolated social situation, non-adherence to medication or on a neuroleptic reduction programme). Exclusion criteria were: learning disabilities, organic brain disease, substance abuse or severe psychotic symptoms. People who were receiving psychotherapy or could not speak English were excluded.

\section{Intervention}

The control group received usual care, comprising medication, psychiatric review and regular follow up with a keyworker. The intervention group received usual care plus 5 sessions of cognitive behavioural therapy during the first 12 weeks and further targeted cognitive behavioural therapy (2 to 3 sessions per week) if early signs of relapse were present (e.g. racing thoughts, suspiciousness; assessed by fortnightly questionnaires or by the treatment team).

\section{Main outcome measures}

Primary outcomes were hospital admission or relapse of schizophrenia.

\section{Main results}

28 people in the intervention group had early signs of relapse and received targeted cognitive behavioural therapy. At 12 months, relapse and hospital admission rates were lower in the intervention group than in the control group (relapse: $18.1 \%$ v 34.7\%; hazard ratio 0.47 , $95 \%$ CI 0.24 to 0.92 , NNT6; hospital admission: $15.3 \%$ v $26.4 \%$; hazard ratio $0.53,95 \%$ CI 0.25 to 1.10 ). However, the result for hospital admission did not reach statistical significance.

\section{Conclusions}

Targeting cognitive behaviour therapy to people with schizophrenia whose prodromal symptoms increase, may reduce relapse rates and hospital admission compared with usual care.

\section{COMMENTARY}

Cognitive behavioural therapy is of considerable topical interest in schizophrenia, despite the fact that the most recent Cochrane meta-analysis provided only qualified support for its effectiveness.

The authors report a large trial (72 patients in each treatment arm) of cognitive-behavioural therapy for relapse prevention in schizophrenia. The study period was 12 months. The trial was randomised, but there was no control intervention; the comparison group received only treatment as usual. Nor were the assessments carried out under blind conditions. In the cognitive behavioural therapy condition, participants were first given a 5-session engagement phase, followed by an intensive targeted intervention (2-3 sessions per week) if and when early signs of relapse appeared. In contrast to previous trials of relapse prevention strategies, medication was not increased. Relapse was defined in two ways, as admission to hospital and as a predetermined increase in positive symptoms.

Relapse as defined by hospitalisation was numerically but not significantly reduced in the cognitive behavioural therapy group (11/72 $v 19 / 72)$. Relapse as defined as by worsening of symptoms was significantly lower in the cognitive behavioural therapy group (13/72 $v$ 25/32).

While the authors suggest that the findings add to the growing evidence for the effectiveness of cognitive behavioural therapy in schizophrenia, their results are marginal and will do little to satisfy the growing numbers of psychiatrists who want to see trials carried out under blind conditions and incorporating measures to control for the non-specific effects of psychological intervention. Peter J McKenna, MA MB ChB MRC Psych

Consultant Psychiatrist Fulbourn Hospital Cambridge, UK

1 Cormac I, Jones C, Campbell C. Cognitive behaviour therapy or schizophrenia (Cochrane Review). In The Cochrane Library, Issue 2. Oxford: Update Software, 2002
Source of funding: This work was funded by the Chief Scientist Office, Scottish Executive.

For correspondence: A Gumley. University of Glasgow, Department of Psychological Medicine, Academic Centre, Garnavel Royal

Hospital, 1055 Great Western Road, Glasgow G12 OXH 